\title{
Catalyst Free One-Pot Synthesis of Chromeno Quinolines and Their Antibacterial Activity
}

\author{
Sruthi Vasamsetty ${ }^{1}$, Sunitha Medidi1,2, Satheesh Ampolu1, Ravi Kumar Majji', \\ Mastan Rao Kotupalli ${ }^{3}$, Chikurumilli China Satyanarayana ${ }^{4}$, Annapurna Nowduri ${ }^{1}$, \\ Paul Douglas Sanasi ${ }^{*}$ \\ ${ }^{1}$ Department of Engineering Chemistry, AU College of Engineering (A), Andhra University, Visakhapatnam, India \\ ${ }^{2}$ SKR College for Women, Rajamahendravaram, India \\ ${ }^{3}$ Department of Chemistry, Dodoma University, Dodoma, Tanzania \\ ${ }^{4}$ SKBR College, Amalapuram, India \\ Email: *spauldouglas.engchem@auvsp.edu.in
}

How to cite this paper: Vasamsetty, S., Medidi, S., Ampolu, S., Majji, R.K., Kotupalli, M.R., Satyanarayana, C.C., Nowduri, A. and Sanasi, P.D. (2017) Catalyst Free One-Pot Synthesis of Chromeno Quinolines and Their Antibacterial Activity. Green and Sustainable Chemistry, 7, 141151.

https://doi.org/10.4236/gsc.2017.72011

Received: March 14, 2017

Accepted: May 19, 2017

Published: May 22, 2017

Copyright $\odot 2017$ by authors and Scientific Research Publishing Inc. This work is licensed under the Creative Commons Attribution International License (CC BY 4.0).

http://creativecommons.org/licenses/by/4.0/

\begin{abstract}
An efficient greener one pot synthesis of dimethyl-dihydro-7H-chromeno[3, 2-h] quinolin- $8(9 \mathrm{H})$-one derivatives has been synthesized through cyclization of aromatic aldehyde, dimidone and 8-hydroxy-quinoline through one-pot condensation method is described. The synthesized compounds are screened for further biological activities against Escherichia coli, Pseudomonas aeruginosa, Staphylococcus aureus, Bacillus subtilis, Bacillus using cut plate method and disc diffusion method.
\end{abstract}

\section{Keywords}

Dimethyl-Dihydro-7H-Chromeno[3,2-h]Quinolin-8(9H)-One Derivatives Synthesis, Biological Activity and One-Pot Condensation

\section{Introduction}

Multi-component reactions (MCRs) have a great role in the organic synthesis. These are one step reactions, where the reactants are subjected into a single reactor, to form a desired product with high yields, without any intermediate formation. Its importance lies mainly in the synthesis of medicinally potent compounds and its convenient preparation than the conventional methods to form privileged scaffolds in a single step process, thereby having great advantage over convergent and conventional synthesis [1] [2] [3].

The product molecules consisting of quinoline and chromene moiety have a broad range of application with biological activity such as anti-malarial, anti-asthmatic, anti-inflammatory and anti-bacterial property [4] [5]. Chromene is 
the privileged structural component for various natural products consisting of photochemical properties. It is the backbone of many polyphenols found mostly in alkaloids, flavanoids, tocopherols and anthocyanins [6]. The chromene derivatives are potential anticancer agents [7].

From the literature survey, taking xanthenes as a reference some of methods have been developed for the synthesis of dimethyl-dihydro-7H-chromeno[3, 2-h]quinolin-8(9H)-one derivatives. Xanthenes are usually prepared using 2naphthol, aldehydes and dimidone as reactants in presence of various catalysts such as Bronsted acidic ionic liquid Triethylamine-bonded sulfonic acid [8], Ionic Liquid Pyrazinium Di (hydrogen sulfate) [9], ionic liquid 1, 3-disulfonicacid imidazolium hydrogensulfate [10], $N, N^{p}$-dibromo- $N, N^{p}$-1, 2-ethane diylbis ( $p$ toluenesulfonamide) [11], 3-sulfobutyl-1-(3-propyltriethoxysilane) imidazolium hydrogen sulfate on silica-coated $\mathrm{Fe}_{3} \mathrm{O}_{4}$ nanoparticles [12] ammonium chloride [13], silica-bonded imidazolium-sulfonic acid chloride [14], ionic liquid sulfonic acid functionalized pyridinium chloride [15], $\mathrm{ZnO}$ nanoparticles [16], ceric ammonium nitrate [17] under solvent free conditions and Bismuth (III) nitrate using water as solvent [18], phosphomolybdic acid using dichloroethane as solvent [19], ceric ammonium nitrate using DCM-ethanol as solvent [20], Thiamine hydrochloride using hexadecyltrimethylammonium bromide (CTAB) in aqueous micellar form as solvent [21] as catalysts.

Though these methods involve their own limitations like longer reaction times [8] [11] [12] [17] using toxic reagents [8] [9] [10] [11] [14] [19] and [21], difficulty to separate the catalyst [13] and [18] and catalyst degradation during the process of the reaction which cannot be recovered [17] [19] and [20]. The main objective of our research is the organic synthesis, which involves green procedures, short reaction time, low temperature conditions, higher yields, and economically desirable processes without any use of catalyst.

In continuation to the synthesis, characterization and catalytic application of nano copper and cobalt ferrite catalysts was reported by us in the preparation of 2, 4, 5,-trisubstitued imidazoles by one-pot synthesis [22], tri and tetra substituted imidazoles under ultrasonication catalyzed by nano copper ferrite [23], microwave assisted synthesis of $\beta$-acetamido ketones, catalyzed by nickel cobalt ferrite [24] poly substituted pyridine derivatives with copper ferrite [25] $4 H$ Pyrano $[3,2-h]$ quinoline derivatives under microwave irradiation with nano cobalt ferrite [26]. New greener reactions paths have been investigated.

Now we report an efficient greener synthesis of dimethyl-dihydro-7H-chromeno [3, 2-h]quinolin-8(9H)-one derivatives through cyclization of aromatic aldehyde, dimidone and 8-hydroxy quinoline (Scheme 1) through one pot condensation method.

\section{Experimental}

\subsection{Chemicals and Apparatus}

All chemicals used in this process are of AR grade fine chemicals, without any further purification. The synthesized dimethyl-dihydro-7H-chromeno [3, 2-h] 


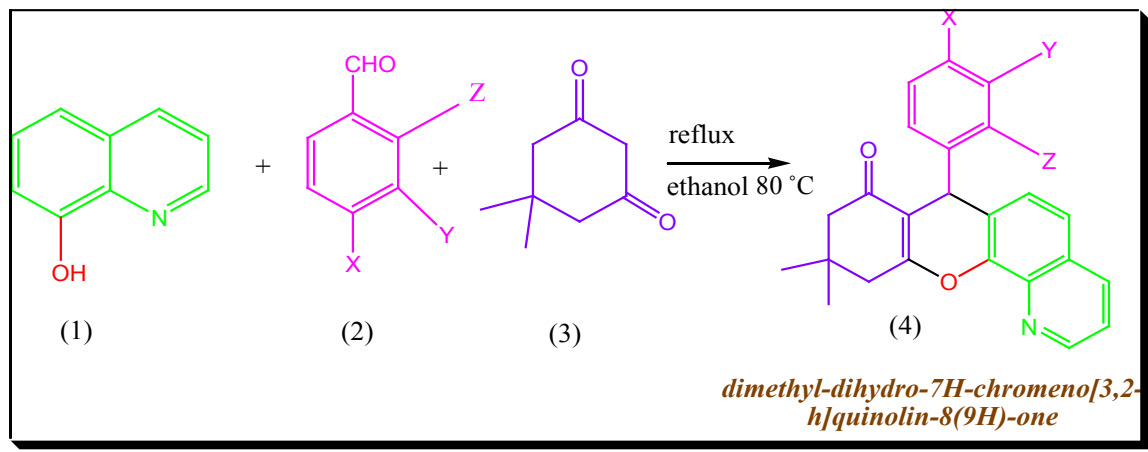

(a) $\mathrm{X}=\mathrm{Cl}, \mathrm{Y}=\mathrm{H}, \mathrm{Z}=\mathrm{H}$; (b) $\mathrm{X}=\mathrm{OH}, \mathrm{Y}=\mathrm{OC}_{2} \mathrm{H}_{5}, \mathrm{Z}=\mathrm{H}$; (c) $\mathrm{X}=\mathrm{OH}, \mathrm{Y}=\mathrm{H} \mathrm{Z}=\mathrm{H}$; (d) $\mathrm{X}=\mathrm{CH}_{3}$, $\mathrm{Y}=\mathrm{H}, \mathrm{Z}=\mathrm{H}$;(e) $\mathrm{X}=\mathrm{H}, \mathrm{Y}=\mathrm{H}, \mathrm{Z}=\mathrm{H}$; (f) $\mathrm{X}=\mathrm{NO}_{2}, \mathrm{Y}=\mathrm{H}, \mathrm{Z}=\mathrm{H}$; (g) $\mathrm{X}=\mathrm{H}, \mathrm{Y}=\mathrm{H}, \mathrm{Z}=\mathrm{NO}_{2}$; (h) $\mathrm{X}=\mathrm{H}, \mathbf{Y}=\mathbf{H}, \mathbf{Z}=\mathrm{OCH}_{3}$

Scheme 1. Synthesis of dimethyl-dihydro-7H-chromeno[3, 2-h]quinolin-8(9H)-one derivatives.

quinolin-8(9H)-one derivatives were characterized by FTIR, MASS and ${ }^{1} \mathrm{H}$ NMR. FTIR spectra recorded on a (Perkin Elmer Spectra-880) spectrophotometer by using $\mathrm{KBr}$ pellets in the region $400-4500 \mathrm{~cm}^{-1}$ and ${ }^{1} \mathrm{H}$ NMR spectra was characterized by $400 \mathrm{MHz}$-(Bruker Avance) in $\mathrm{CDCl}_{3}$ solvent and MASS spectra was recorded at $70 \mathrm{eV}$ (MASPEC low resolution mass spectrometer).

\subsection{General Procedure for the Synthesis of Dimethyl-Dihydro-7H-Chromeno [3, 2-h]Quinolin-8(9H)-One Derivatives}

The one pot synthesis of dimethyl-dihydro-7H-chromeno [3, 2-h]quinolin$8(9 \mathrm{H})$-one derivatives was carried out in $250 \mathrm{~mL}$ round bottomed flask by taking Equimolar quantities of aromatic aldehydes $(10 \mathrm{mmol})$, dimidone $(10 \mathrm{mmol})$ and 8-hydoxyquinoline $(10 \mathrm{mmol})$ and $15 \mathrm{~mL}$ of ethanol were mixed together and the flask was placed in oil bath over a hotplate consisting of magnetic stirrer and kept for reflux at $80^{\circ} \mathrm{C}$. The progress of the reaction was monitored by TLC using mobile phase (n-Hexane: ethyl acetate 3:1). The formed product mixture was cooled to room temperature and ethyl alcohol added until the product was dissolved. The products were recrystalized with ethanol and characterized and compared by FT-IR, ${ }^{1} \mathrm{H}$ NMR and MASS spectral techniques are tabulated in Table 1.

\section{Results and Discussion}

The procedure involves the cyclization of aromatic aldehyde, dimidone and 8 -hydoxyquinoline is described as model reaction shown in Scheme 1. The attainability of formation of chromeno [3, 2-h] quinolin-8(9H)-one derivatives and the reaction conditions are tabulated in Table 2.

\subsection{Comparative Study for the Synthesis of Dimethyl-Dihydro-7H-Chromeno [3, 2-h]Quinolin-8(9H)-One Derivatives with Other Catalysts}

Reaction times for the formation of chromeno[3, 2-h] quinolin-8(9H)-one derivatives with various catalysts are presented in Table 3 . It is observed that with 
Table 1. Reactants and spectral data of oxazino quinoline derivatives.

S.No Reactants dimethyl-dihydro-7H-chromeno [3, 2-h]quinolin-8(9H)-one

7-(4-chlorophenyl)-10, 10-dimethyl-10, 11-dihydro-7 $H$-chromeno[3, 2- $h]$ quinolin-8(9H)-one (4a)

Pale White solid, yield 94\% IR ( $\left.\mathrm{KBr}, v_{\max } \mathrm{cm}^{-1}\right): 2900$ (CH str), 1640 (-C=C str), 1450 (-C-C= str), 1250 (-C-O-C str); ${ }^{1} \mathrm{H}$ NMR $\left(\mathrm{CDCl}_{3}-400 \mathrm{MHz}, \delta \mathrm{ppm}\right) ; 8.9(\mathrm{~m}, \mathrm{Ar}-\mathrm{H}), 8.7$ (m, Ar-H), 7.34 (m, Ar-H), 7.26 (m, Ar-H), 7.17 (m, Ar-H), 7.01 (m, Ar-H), $6.29(\mathrm{~m}$, Ar-H), 3.9 (s, 1H), $2.34(\mathrm{~s}, 1 \mathrm{H}) 1.08$ (s, methyl proton), 1.03 (s, methyl proton); ESMS: $389.2[\mathrm{M}+1]$.

2.<smiles>Oc1cccc2cccnc12</smiles><smiles>CCOc1cc(C=O)ccc1O</smiles><smiles>CCOc1cc(C2C3=C(CC(C)(C)CC3=O)Oc3c2ccc2cccnc32)ccc1O</smiles>

7-(3-ethoxy-4-hydroxyphenyl)-10, 10-dimethyl-10, 11-dihydro-7H-chromeno[3, 2-h]quinolin-8(9H)-one (4b)

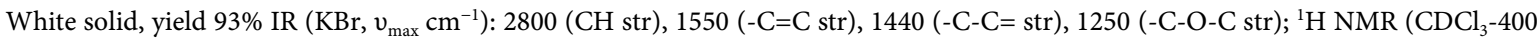
MHz, $\delta$ ppm); 8.99 (m, Ar-H), 8.17 - 8.11 (m, Ar-H), 7.47 - 7.41 (m, Ar-H), 7.39 - 7.32 (m, Ar-H), 7.19 - 7.10 (m, Ar-H), 7.08 - 7.03 $(\mathrm{m}, \operatorname{Ar}-\mathrm{H}), 6.85-6.83(\mathrm{~m}, \mathrm{Ar}-\mathrm{H}), 6.79-6.71(\mathrm{~m}, \mathrm{Ar}-\mathrm{H}), 5.2\left(\mathrm{~s}\right.$, hydroxy H), $4.21(\mathrm{~s}, 1 \mathrm{H}), 4.0\left(\mathrm{q}, 2 \mathrm{H}, \mathrm{CH}_{2}\right.$ proton), $2.8(\mathrm{~s}, 1 \mathrm{H}), 2.3(\mathrm{~s}$, $1 \mathrm{H}), 1.3$ (t, methyl proton), 1.1 (s, methyl proton); ESMS: $416.1[\mathrm{M}+1]$.<smiles>Oc1cccc2cccnc12</smiles><smiles>O=Cc1ccc(O)cc1</smiles><smiles>CC1(C)CC(=O)CC(=O)C1</smiles><smiles>CC1(C)CC(=O)C2=C(C1)Oc1c(ccc3cccnc13)C2c1ccc(O)cc1</smiles>

7-(4-hydroxyphenyl)-10, 10-dimethyl-10, 11-dihydro-7H-chromeno[3, 2-h]quinolin-8(9H)-one (4c) Pale yellow solid, yield 89\% IR ( $\left.\mathrm{KBr}, v_{\max } \mathrm{cm}^{-1}\right): 2900$ (CH str), 1640 (-C=C str), 1500 (-C-C= str), 1200 (-C-O-C str); ${ }^{1} \mathrm{H}$ NMR $\left(\mathrm{CDCl}_{3}-400 \mathrm{MHz}, \delta \mathrm{ppm}\right) ; 8.86$ (m, Ar-H), 8.04 (m, Ar-H), 7.8 (m, Ar-H), 7.5 (m, Ar-H), 7.12 (m, Ar-H), 6.80 - 6.80 (m, Ar-H), 6.36 $(\mathrm{m}, \mathrm{Ar}-\mathrm{H}), 5.0(\mathrm{~s}$, hydroxy H), $4.5(\mathrm{~s}, 1 \mathrm{H}), 2.8(\mathrm{~s}, 1 \mathrm{H}), 2.0(\mathrm{~s}, 1 \mathrm{H}), 1.3$ (s, methyl proton), 1.0 (s, methyl proton); ESMS: 372 [M + 1].

4.<smiles>Oc1cccc2cccnc12</smiles><smiles>Cc1ccc(C=O)cc1</smiles><smiles>CC1(C)CC(=O)CC(=O)C1</smiles><smiles>Cc1ccc(C2C3=C(CC(C)(C)CC3=O)Oc3c2ccc2cccnc32)cc1</smiles>

10, 10-dimethyl-7-p-tolyl-10, 11-dihydro-7H-chromeno[3, 2-h]quinolin-8(9H)-one (4d)

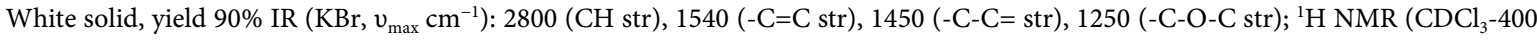
MHz, $\delta$ ppm); 8.80 - 8.87 (m, Ar-H), 8.21 - 8.19 (m, Ar-H), 7.26 (m, Ar-H), 7.15 (m, Ar-H), 7.14 (m, Ar-H), 7.08 - 7.04 (m, Ar-H), 6.2 (s, 1H), $2.4(\mathrm{~s}, 1 \mathrm{H}), 2.35$ (s, methyl proton), $2.1(\mathrm{~s}, 1 \mathrm{H}), 1.10$ (s, methyl proton), 1.04 (s, methyl proton); ESMS: 370.3 [M + 1]. 


\section{Continued}<smiles>Oc1cccc2cccnc12</smiles><smiles>O=Cc1ccccc1</smiles><smiles>CC1(C)CC(=O)CC(=O)C1</smiles><smiles>CC1(C)CC(=O)C2=C(C1)Oc1c(ccc3cccnc13)C2c1ccccc1</smiles>

10, 10-dimethyl-7-phenyl-10, 11-dihydro-7H-chromeno[3, 2-h]quinolin-8(9H)-one(4e)

Pale White solid, yield 88\% IR ( $\left.\mathrm{KBr}, v_{\max } \mathrm{cm}^{-1}\right): 2800$ (CH str), 1593.27 (-C=C str), 1440 (-C-C= str), 1050 (-C-O-C str); ${ }^{1} \mathrm{H}$ NMR $\left(\mathrm{CDCl}_{3}-400 \mathrm{MHz}, \delta \mathrm{ppm}\right) ; 8.8$ (m, Ar-H), 8.1 (m, Ar-H), 7.29 (m, Ar-H), 7.27 - 7.25 (m, Ar-H), 7.19 (m, Ar-H), 7.17 (m, Ar-H), 7.11 7.09 (m, Ar-H), 5.55 (s, 1H), $2.49-2.39$ (s, 1H), $2.34-2.29$ (s, 1H), 1.24 (s, methyl proton), 1.10 (s, methyl proton); ESMS: 356.3 [M + $1]$.<smiles>Oc1cccc2cccnc12</smiles><smiles>O=Cc1ccc([N+](=O)[O-])cc1</smiles><smiles>CC1(C)CC(=O)CC(=O)C1</smiles><smiles>CC1(C)CC(=O)C2=C(C1)Oc1c(ccc3cccnc13)C2c1ccc([N+](=O)[O-])cc1</smiles>

10, 10-dimethyl-7-(4-nitrophenyl)-10, 11-dihydro-7H-chromeno[3, 2-h]quinolin-8(9H)-one(4f)

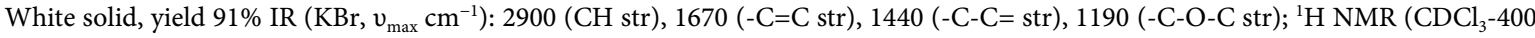
MHz, $\delta \mathrm{ppm}) ; 8.86$ (m, Ar-H), 8.25 (m, Ar-H), 8.18 (m, Ar-H), 7.54 (m, Ar-H), 7.44 - 7.42 (m, Ar-H), 7.26 (m, Ar-H), 7.0 (m, Ar-H), $6.2(\mathrm{~s}, 1 \mathrm{H}), 2.36(\mathrm{~s}, 1 \mathrm{H}), 1.56(\mathrm{~s}, 1 \mathrm{H}), 1.10(\mathrm{~s}$, methyl proton), 1.03 (s, methyl proton); ESMS: $401.9[\mathrm{M}+1]$.<smiles>Oc1cccc2cccnc12</smiles><smiles>O=Cc1ccccc1[N+](=O)[O-]</smiles><smiles>CC1(C)CC(=O)CC(=O)C1</smiles><smiles>CC1(C)CC(=O)C2=C(C1)Oc1c(ccc3cccnc13)C2c1ccccc1[N+](=O)[O-]</smiles>

10, 10-dimethyl-7-(2-nitrophenyl)-10, 11-dihydro-7H-chromeno[3, 2-h]quinolin-8(9H)-one(4g)

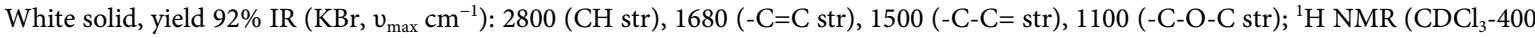
$\mathrm{MHz}, \delta \mathrm{ppm}) ; 8.86(\mathrm{~m}, \mathrm{Ar}-\mathrm{H}), 8.00(\mathrm{~m}, \mathrm{Ar}-\mathrm{H}), 7.98(\mathrm{~m}, \mathrm{Ar}-\mathrm{H}), 7.56$ - $7.54(\mathrm{~m}, \mathrm{Ar}-\mathrm{H}), 7.49$ - 7.45 (m, Ar-H), 7.47 - 7.40 (m, Ar-H), 7.38 - 7.35 (m, Ar-H), 7.33 - 7.31 (m, Ar-H), 7.29 - 7.24 (m, Ar-H), $5.02(\mathrm{~s}, 1 \mathrm{H}), 2.48$ - $2.45(\mathrm{~m}, 1 \mathrm{H}), 2.33$ - $2.26(\mathrm{~m}, 1 \mathrm{H}), 1.12(\mathrm{~s}, \mathrm{me}-$ thyl proton), $1.05-1.02$ (s, methyl proton); ESMS: $401.2[\mathrm{M}+1]$.

8.<smiles>Oc1cccc2cccnc12</smiles><smiles>COc1ccccc1C=O</smiles><smiles>CC1(C)CC(=O)CC(=O)C1</smiles><smiles>COc1ccccc1C1C2=C(CC(C)(C)CC2=O)Oc2c1ccc1cccnc21</smiles>

7-(2-methoxyphenyl)-10, 10-dimethyl-10, 11-dihydro-7H-chromeno[3, 2-h]quinolin-8(9H)-one(4h)

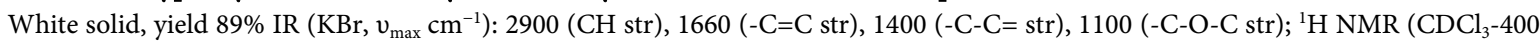
MHz, $\delta$ ppm); 8.79 - 8.77 (m, Ar-H), 8.19 - 8.17 (m, Ar-H), 7.41 - 7.40 (m, Ar-H), 7.39 - 7.27 (m, Ar-H), 7.06 - 7.04 (m, Ar-H), 7.03 $7.01(\mathrm{~m}, \mathrm{Ar}-\mathrm{H}), 6.94-6.92(\mathrm{~m}, \mathrm{Ar}-\mathrm{H}), 6.51(\mathrm{~m}, \mathrm{Ar}-\mathrm{H}), 4.9(\mathrm{~s}, 1 \mathrm{H}), 3.73$ (s, methoxy proton) $2.3(\mathrm{~m}, 1 \mathrm{H}), 2.27(\mathrm{~m}, 1 \mathrm{H}), 1.09$ (s, methyl proton), 1.06 (s, methyl proton); ESMS: $386.2[\mathrm{M}+1]$. 
Table 2. Synthesis of dimethyl-dihydro-7H-chromeno [3, 2-h]quinolin-8(9H)-one derivatives.

\begin{tabular}{|c|c|c|c|c|c|c|}
\hline S. no & $\mathrm{X}$ in aldehyde & $\mathrm{Y}$ in aldehyde & $\mathrm{Z}$ in aldehyde & Product & Time (min) & Yield \\
\hline 1 & $\mathrm{Cl}$ & $\mathrm{H}$ & $\mathrm{H}$ & $4 \mathrm{a}$ & 30 & 94 \\
\hline 2 & $\mathrm{OH}$ & $\mathrm{OC}_{2} \mathrm{H}_{5}$ & $\mathrm{H}$ & $4 b$ & 35 & 93 \\
\hline 3 & $\mathrm{OH}$ & $\mathrm{H}$ & $\mathrm{H}$ & $4 c$ & 30 & 89 \\
\hline 4 & $\mathrm{CH}_{3}$ & $\mathrm{H}$ & $\mathrm{H}$ & $4 d$ & 40 & 90 \\
\hline 5 & $\mathrm{H}$ & $\mathrm{H}$ & $\mathrm{H}$ & $4 \mathrm{e}$ & 35 & 88 \\
\hline 6 & $\mathrm{NO}_{2}$ & $\mathrm{H}$ & $\mathrm{H}$ & $4 \mathrm{f}$ & 40 & 91 \\
\hline 7 & $\mathrm{H}$ & $\mathrm{H}$ & $\mathrm{NO}_{2}$ & $4 \mathrm{~g}$ & 40 & 92 \\
\hline 8 & $\mathrm{H}$ & $\mathrm{H}$ & $\mathrm{OCH}_{3}$ & $4 \mathrm{~h}$ & 30 & 89 \\
\hline
\end{tabular}

Table 3. Comparative study of synthesis with catalysts.

\begin{tabular}{|c|c|c|c|c|c|}
\hline S. No & Catalyst & Solvent used & Time (min) & Yield & Ref No \\
\hline 1 & Triethylamine-bonded sulfonic acid & Solvent free & 30 (reflux) & 94 & [7] \\
\hline 2 & $N, N^{p}$-dibromo- $N, N^{p}$-1, 2-ethanediylbis ( $p$-toluenesulfonamide) & Solvent free & 120 (reflux) & 92 & [10] \\
\hline 3 & ceric ammonium nitrate & Ethyl acetate & 135 (ultrasonication) & 87 & [16] \\
\hline 4 & $\begin{array}{l}\text { 3-sulfobutyl-1-(3-propyltriethoxysilane) imidazolium hydrogen } \\
\text { sulfate on silica-coated } \mathrm{Fe}_{3} \mathrm{O}_{4} \text { nanoparticles }\end{array}$ & Ethyl acetate & 30 (reflux) & 90 & [11] \\
\hline
\end{tabular}

other catalysts the reactions times are very much higher. Under reflux conditions, synthesis of dimethyl-dihydro-7H-chromeno [3, 2-h]quinolin-8(9H)-one derivatives without use of any catalyst has been reported. The present method offers a comparatively very low cost and easy preparation.

\subsection{Plausible Mechanism for the Synthesis of Dimethyl-Dihydro-7H-Chromeno [3, 2-h]Quinolin-8(9H)-One Derivatives}

In this reaction 8-hyroxy quinoline, aldehydes and dimidone are taken as reactants to run the process. Initially aromatic aldehydes undergo nucleophillic addition with 8-hydroxy quinoline through knovenegal condensation mechanism to form the intermediate Knovenegal product (1). In the second step the dimidone undergo enolisation. The formed enol product reacts with knovenegal product to form the highly stabilized product dimethyl-dihydro-7H-chromeno [3, 2-h]quinolin-8(9H)-one derivatives smoothly shown in Scheme 2.

\section{Biological Activity}

The antibiotic potency can be determined using the microbial assays. The basic principle of microbial assay lies in comparison of the inhibition of growth of bacteria by measuring concentration of the product to be investigated with that produced by known concentration of the antibiotic having a known activity.

The methods used for assay are cup plate method and disc diffusion method. The cup plate method is based on the diffusion of an antibiotic from a cavity 
<smiles>C=C=CC1(C)CC(=O)CC(=O)C1</smiles>

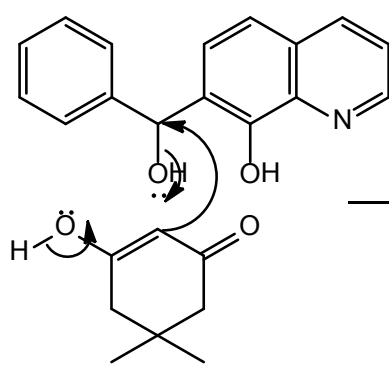

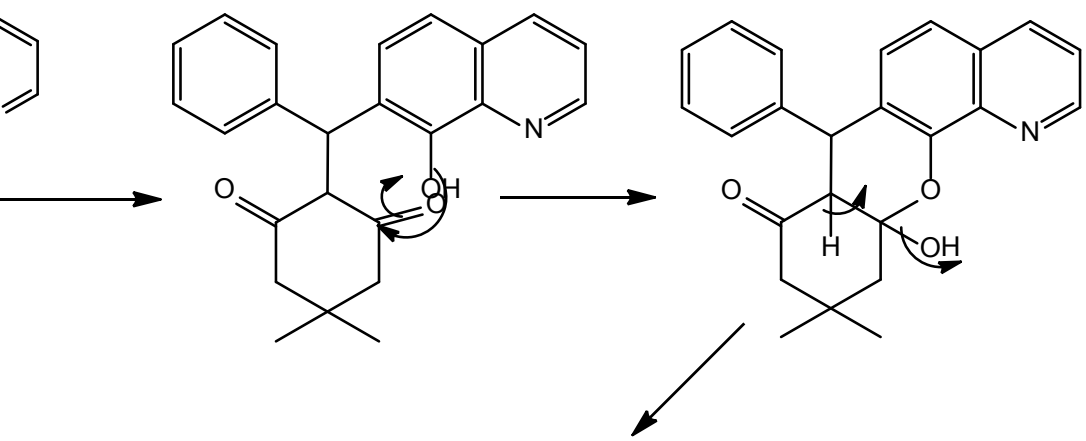<smiles>CC1(C)CC(=O)C2=C(C1)Oc1c(ccc3cccnc13)C2c1ccccc1</smiles>

Scheme 2. Plausible mechanism of dimethyl-dihydro-7H-chromeno[3, 2-h] quinolin-8(9H)-one derivatives.

through the solidified agar layer of a Petri-dish. Growth of inoculated microbe is inhibited entirely in a circular zone around a cavity containing a solution of the antibiotics. Antimicrobial activity of synthesized compounds was screened against four human pathogenic bacteria, two gram positive and two gram negative bacteria and their respective MTCCNO numbers are given in parenthesis as Escherichia coli (Gram -ve)-(2692), Pseudomonas aeruginosa (Gram -ve)(2453), Staphylococcus aureus (Gram +ve)-(902), Bacillus subtilis (Gram +ve)(441). The activities of the drug samples against 4 human pathogenic bacteria are tabulated in Table 4.

The antibacterial activity of the samples is assessed using the different concentration of the sample i.e., low, intermediate, high.

The present investigation reveals that the zone of inhibition increased as the concentration of the sample increased. This is seen in case of the compounds $4 \mathrm{a}$ and $4 \mathrm{e}, 4 \mathrm{~h}$ Hence the MIC (Minimum Inhibitory Concentration) of these samples that can inhibit bacterial growth is $10 \mu \mathrm{l}, 20 \mu \mathrm{l}$ and $30 \mu \mathrm{l}$ respectively. Thus the above samples are able to show antibacterial activity on Escherichia coli, 
Table 4. Antibacterial activities of drug samples.

\begin{tabular}{|c|c|c|c|c|c|}
\hline \multirow{3}{*}{$\begin{array}{l}\text { SERIAL } \\
\text { NUMBER }\end{array}$} & \multirow{3}{*}{ SAMPLE } & \multirow{3}{*}{ MICROORGANISM } & \multirow{2}{*}{\multicolumn{3}{|c|}{$\begin{array}{c}\text { Concentration of the sample } \\
\text { Zone of inhibition (mm) }\end{array}$}} \\
\hline & & & & & \\
\hline & & & $10 \mu \mathrm{l}$ & $20 \mu \mathrm{l}$ & $30 \mu \mathrm{l}$ \\
\hline \multirow[t]{5}{*}{1} & $4 \mathrm{a}$ & Escherichia coli & V3.0 & V3.1 & V3.4 \\
\hline & & Pseudomonas aeruginosa & V3.0 & V3.1 & V3.2 \\
\hline & & Staphylococcus aureus & V3.1 & V3.2 & V3.4 \\
\hline & & Bacillus subtilis & V3.1 & V3.4 & V3.6 \\
\hline & & & $10 \mu \mathrm{l}$ & $20 \mu \mathrm{l}$ & $30 \mu \mathrm{l}$ \\
\hline \multirow[t]{5}{*}{2} & $4 \mathrm{~b}$ & Escherichia coli & $\mathrm{V} 2.7$ & V3.0 & V3.2 \\
\hline & & Pseudomonas aeruginosa & V3.1 & V3.2 & V3.4 \\
\hline & & Staphylococcus aureus & V1.9 & V2.5 & V3.0 \\
\hline & & Bacillus subtilis & V3.0 & V3.2 & V3.7 \\
\hline & & & $10 \mu \mathrm{l}$ & $20 \mu \mathrm{l}$ & $30 \mu \mathrm{l}$ \\
\hline \multirow[t]{5}{*}{3} & $4 c$ & Escherichia coli & V1.7 & V2.0 & V2.5 \\
\hline & & Pseudomonas aeruginosa & V2.0 & V2.5 & V2.7 \\
\hline & & Staphylococcus aureus & $\mathrm{V} 2.2$ & V2.4 & $\mathrm{V} 2.7$ \\
\hline & & Bacillus subtilis & V2.4 & $\mathrm{V} 2.5$ & $\mathrm{~V} 2.7$ \\
\hline & & & $10 \mu \mathrm{l}$ & $20 \mu \mathrm{l}$ & $30 \mu \mathrm{l}$ \\
\hline \multirow[t]{5}{*}{4} & $4 \mathrm{~d}$ & Escherichia coli & V1.1 & V1.2 & V1.4 \\
\hline & & Pseudomonas aeruginosa & V & V1.7 & V1.9 \\
\hline & & Staphylococcus aureus & V2.4 & $\mathrm{V} 2.7$ & V3.2 \\
\hline & & Bacillus subtilis & - & - & - \\
\hline & & & $10 \mu \mathrm{l}$ & $20 \mu \mathrm{l}$ & $30 \mu \mathrm{l}$ \\
\hline \multirow[t]{5}{*}{5} & $4 e$ & Escherichia coli & V3.7 & V4.0 & V4.7 \\
\hline & & Pseudomonas aeruginosa & V3.0 & V3.5 & V4.0 \\
\hline & & Staphylococcus aureus & $\mathrm{V} 2.3$ & V2.5 & $\mathrm{V} 2.7$ \\
\hline & & Bacillus subtilis & V3.2 & V3.5 & V3.7 \\
\hline & & & $10 \mu \mathrm{l}$ & $20 \mu \mathrm{l}$ & $30 \mu \mathrm{l}$ \\
\hline \multirow[t]{5}{*}{6} & $4 \mathrm{f}$ & Escherichia coli & V2.5 & V3.0 & V3.5 \\
\hline & & Pseudomonas aeruginosa & $\mathrm{V} 2.0$ & V2.5 & V3.0 \\
\hline & & Staphylococcus aureus & V3.0 & V3.5 & V4.0 \\
\hline & & Bacillus subtilis & V2.5 & V3.0 & V3.7 \\
\hline & & & $10 \mu \mathrm{l}$ & $20 \mu \mathrm{l}$ & $30 \mu \mathrm{l}$ \\
\hline \multirow[t]{5}{*}{7} & $4 \mathrm{~g}$ & Escherichia coli & V3.1 & V3.2 & V3.4 \\
\hline & & Pseudomonas aeruginosa & $\mathrm{V} 2.3$ & V2.5 & $\mathrm{V} 2.7$ \\
\hline & & Staphylococcus aureus & $\mathrm{V} 2.0$ & V2.5 & V3.0 \\
\hline & & Bacillus subtilis & - & - & - \\
\hline & & & $10 \mu \mathrm{l}$ & $20 \mu \mathrm{l}$ & $30 \mu \mathrm{l}$ \\
\hline \multirow[t]{5}{*}{8} & $4 \mathrm{~h}$ & Escherichia coli & V3.0 & V3.2 & V3.5 \\
\hline & & Pseudomonas aeruginosa & $\mathrm{V} 2.2$ & V2.7 & V3.5 \\
\hline & & Staphylococcus aureus & V2.5 & V3.0 & V3.5 \\
\hline & & Bacillus subtilis & $\mathrm{V} 2.5$ & V3.6 & V4.0 \\
\hline & & & $10 \mu \mathrm{l}$ & $20 \mu \mathrm{l}$ & $30 \mu \mathrm{l}$ \\
\hline \multirow[t]{4}{*}{9} & Standard Streptomycin & Escherichia coli & V 3.0 & V3.1 & V3.4 \\
\hline & & Pseudomonas aeruginosa & V3.0 & V3.1 & V3.2 \\
\hline & & Staphylococcus aureus & V3.1 & V3.2 & V3.4 \\
\hline & & Bacillus subtilis & V3.1 & V3.4 & V3.6 \\
\hline
\end{tabular}




\section{Pseudomonas aeruginosa, Staphylococcus aureus, Bacillus subtilis.}

The standard drug streptomycin is found to be very effective anti-microbial agent. Here it is found that the standard drug show antibacterial activity on both Gram +ve and -ve bacteria and it is found that the zone of inhibition increased as the concentration of the sample increased.

\section{Conclusion}

In this present study, we report an efficient greener method for the synthesis of dimethyl-dihydro-7H-chromeno[3, 2-h] quinolin-8(9H)-one derivatives. This method has several advantages like improved yield of products, less reaction times.

\section{References}

[1] Ramon, D.J. and Miguel, Y. (2005) Asymmetric Multicomponent Reactions (AMCRs): The New Frontier. Angewandte Chemie International Edition, 44, 1602-1634. https://doi.org/10.1002/anie.200460548

[2] Domling A. (2006) Recent Developments in Isocyanide Based Multicomponent Reactions in Applied Chemistry. Chemical Reviews, 106, 17-89. https://doi.org/10.1021/cr0505728

[3] Tu, S., Jiang, B., Zhang, Y., Jia, R., Zhang, J., Yao, C. and Feng, S. (2007) An Efficient and Chemoselective Synthesis of N-Substituted 2-Aminopyridines via a Micro-Wave Assisted Multicomponent Reaction. Organic \& Biomolecular Chemistry, 5, 355-359. https://doi.org/10.1039/B614747J

[4] Larsen, R.D., Corley, E.G., King, A.O., Carrol, J.D., Davis, P., Verhoeven, T.R., Reider, P.J., Labelle, M., Gauthier, J.Y., Xiang, Y.B. and Zamboni, R.J. (1996) Practical Route to a New Class of $\mathrm{LTD}_{4}$ Receptor Antagonists. The Journal of Organic Chemistry, 61, 3398-3405. https://doi.org/10.1021/jo952103j

[5] Chen, Y.L., Fang, K.C., Shen, J.Y., Hsu, S.L. and Tzeng, C.C. (2001) Synthesis and Antibacterial Evaluation of Certain Quinolone Derivatives. Journal of Medicinal Chemistry, 44, 2374-2377. https://doi.org/10.1021/jm0100335

[6] Qiao, R., Woon, Y.S., Zhiyun, D., Kun, Z. and Jian, W. (2011) Expeditious Assembly of a 2-Amino-4H-Chromene Skeleton by Using an Enantioselective Mannich Intramolecular Ring Cyclization-Tautomerization Cascade Sequence. ChemistryA European Journal, 17, 7781-7785. https://doi.org/10.1002/chem.201100927

[7] Vosooghi, M., Rajabalian, S., Sorkhi, M., Badinloo, M., Nakhjiri, M. and Negahbani, A.S. (2010) Synthesis and Cytotoxic Activity of Some 2-Amino-4-Aryl-3-Cyano-7(Dimethylamino)-4H-Chromenes. Research in Pharmaceutical Sciences, 5, 9-14.

[8] Abdolkarim, Z., Roghayyeh, K., Maria, M., Masoud, K.R., Mohammad, M.A.Z., Ahmad, R.M.Z. and Alireza, H. (2012) Triethylamine-Bonded Sulfonic Acid $\left\{\left[\mathrm{Et}_{3} \mathrm{~N}\right.\right.$ $\left.\left.\mathrm{SO}_{3} \mathrm{H}\right] \mathrm{Cl}\right\}$ as an Homogeneous Catalyst for the Synthesis of 12-Aryl-8,9,10,12-Tetrahydrobenzo[a]Xanthen-11-Ones. Iranian Journal of Catalysis, 2, 107-114.

[9] Abdolkarim, Z., Roghayyeh, K., Marzieh, H., Mohammad, M., Mohammad, A.Z., Ahmad, R.M.Z., Alireza, H., Ardeshir, K. and Vahid, K. (2012) Efficient Synthesis of 12-Aryl-8,9,10,12-Tetrahydrobenzo[a]-Xanthen-11-Ones Using Ionic Liquid Pyrazinium $\mathrm{Di}\left(\mathrm{Hydrogen}\right.$ Sulfate) $\left\{\mathrm{Py}\left(\mathrm{HSO}_{4}\right)_{2}\right\}$ as a Novel, Green and Homogeneous Catalyst. Journal of Mexican Chemical Society, 56, 389-394.

[10] Farhad, S., Asieh, Y. and Kamal, M. (2014) One-Pot Synthesis of Various Xanthene Derivatives Using Ionic Liquid 1,3-Disulfonic Acid Imidazolium Hydrogen Sulfate 
as an Efficient and Reusable Catalyst under Solvent-Free Conditions. Chinese Chemical Letters, 25, 341-347.

[11] Ramin, G.V. and Seyedeh, M.M. (2010) Facile One-Pot Synthesis of Tetrahydrobenzo[a]Xanthene-11-One and Aryl-14H-Dibenzo[a.j]Xanthene. Organic Preparations and Procedures International, 42, 494-498. https://doi.org/10.1080/00304948.2010.514802

[12] Zhang, Q., Su, H., Luo, J. and Wei, Y. (2012) A Magnetic Nanoparticle Supported Dual Acidic Iionic Liquid: A “Quasi-Homogeneous" Catalyst for the One-Pot Synthesis of Benzoxanthenes. Green Chemistry, 14, 201. https://doi.org/10.1039/C1GC16031A

[13] Naser, F., Akbar, M., Hassan, M., Reza, M. and Hamid, R.M.E. (2011) Ammonium Chloride Catalysed-One-Pot Synthesis Tetrahydrobenzo[ $\alpha]$-Xanthen-11-Ones Derivatives under Solvent Free Conditions. Synthetic Communications, 41, 2663-2673. https://doi.org/10.1080/00397911.2010.515340

[14] Ahmad, R.M.Z., Mohammad, A.Z., Mahmoud, Z., Abdolkarim, Z. and Vahid, K. (2015) Application of Silica-Bonded Iimidazolium-Sulfonic Acid Chloride (SBISAC) as a Heterogeneous Nanocatalyst for the Domino Condensation of Arylaldehydes with 2-Naphthol and Dimedone. Journal of Molecular Liquids, 211, 373-380.

[15] Ahmad, R.M.Z., Mohammad, A.Z., Mahmoud, Z., Abdolkarim, Z. and Vahid, K. (2013) Preparation, Characterization and Application of Ionic Liquid Sulfonic Acid Functionalized Pyridinium Chloride as an Efficientcatalyst for the Solvent-Free Synthesis of 12-Aryl-8,9,10,12-Tetrahydrobenzo[a]-Xanthen-11-Ones. Journal of Molecular Liquids, 186, 63-69.

[16] Javad, S.G. and Mohammad, A.G. (2014) A Simple and Efficient Synthesis of 12-Aryl-8,9,10, 12-Tetrahydrobenzo[a]Xanthen-11-Ones by ZnO Nanoparticles Catalyzed Three Component Coupling Reaction of Aldehydes, 2-Naphthol and Dimedone. South African Journal of Chemistry, 67, 27-32.

[17] ,Atul, K., Siddharth, S., Ram, A.M. and Jayant, S. (2010) Diversity Oriented Synthesis of Benzoxanthene and Benzochromene Libraries via One-Pot, Three-Component Reactions and Their Anti-Proliferative Activity. Journal of Combinatorial Chemistry, 12, 20-24. https://doi.org/10.1021/cc900143h

[18] Bi, B.F.M., Abdolhamid, B. and Naimeh, S. (2012) Synthesis of Tetrahydrobenzo[a] Xanthenes-11-One Derivatives in Water Promoted by $\mathrm{Bi}\left(\mathrm{NO}_{3}\right)_{3} \cdot 5 \mathrm{H}_{2} \mathrm{O}$. CHEMIJA, 23, 118-123.

[19] Srihari, P., Ganganna, B., Rajendra, P.K., Dinesh, C.B. and Yadav, J.S. (2011) Bronsted Acid Catalyzes One-Pot Condensation of b-Naphthol, Aldehyde and Active Methylene Substrate: Synthesis of Napthopyrans. Indian Journal of Chemistry, 50B, 1755-1761.

[20] Sudha, S. and Pasha, M.A. (2012) Ultrasound Assisted Synthesis of Tetrahydrobenzo[c]Xanthene-11-Ones Using CAN as Catalyst. Ultrasonics Sonochemistry, 19, 994-998.

[21] Shahin, F., Pravin, K., Singh, Preyas A., Shireen, Mandavi, S. and Singh, J. (2013) Thiamine Hydrochloride as a Promoter for the Efficient and Green Synthesis of 12-Aryl-8,9,10,12 Tetrahydrobenzoxanthene-11-One Derivatives in Aqueous $\mathrm{Mi}$ cellar Medium. Tetrahedron Letters, 54, 6732-6736.

[22] Sanasi, P.D., Santhipriya, D., Ramesh, Y., Ravi, K.M., Swathi, B. and Jaya Rao, K. (2014) Nano Copper and Cobalt Ferrites as Heterogeneous Catalyst for the One-Pot Synthesis of 2,4,5-Tri Substituted Imidazoles. Journal of Chemical Sciences, 126, 1715-1720. https://doi.org/10.1007/s12039-014-0729-2

[23] Sanasi, P.D., Majji, R.K., Bandaru, S., Bassa, S., Pinninti, S., Vasamsetty, S. and Ko- 
rupolu, R.B. (2016) Nano Copper Ferrite Catalyzed Sonochemical, One-Pot Three and Four Component Synthesis of Poly Substituted Imidazoles. Modern Research in Catalysis, 5, 31-44. https://doi.org/10.4236/mrc.2016.51004

[24] Sanasi, P.D., Chilla, P.N., Ramesh, Y., Satyanarayana, B., Majji, R.K., Bandaru, S., Jeldi, R.K. and Vasamsetty, S. (2015) Microwave Assisted Nickel Cobalt Ferrite Catalyzed One-Pot Synthesis of $\beta$-Acetamido Ketones. International Journal of Engineering Science and Technology, 7, 418-425.

[25] Sanasi, P.D., Bandaru, S., Majji, R.K., Ramesh, Y., Jaya Rao, K., Satyanarayana, B. and Chilla, P.N. (2016) Nano Copper Ferrite Catalyzed Improved Procedure for One-Pot Synthesis of Poly Substituted Pyridine Derivatives. Chemical Science Transactions, 5, 325-334.

[26] Swathi, B., Ravi, K.M., Satyanarayana, B., Pandu, N.C., Ramesh, Y., Sruthi, V., Rajendra, K.J., Raghu, B.K. and Paul, D.S. (2016) Magnetic Nano Cobalt Ferrite Catalyzed Synthesis of $4 H$-Pyrano[3,2- $h$ ] Quinoline Derivatives under Microwave Irradiation. Green and Sustainable Chemistry, 6, 101-109.

https://doi.org/10.4236/gsc.2016.62009

Submit or recommend next manuscript to SCIRP and we will provide best service for you:

Accepting pre-submission inquiries through Email, Facebook, LinkedIn, Twitter, etc. A wide selection of journals (inclusive of 9 subjects, more than 200 journals)

Providing 24-hour high-quality service

User-friendly online submission system

Fair and swift peer-review system

Efficient typesetting and proofreading procedure

Display of the result of downloads and visits, as well as the number of cited articles

Maximum dissemination of your research work

Submit your manuscript at: http://papersubmission.scirp.org/

Or contact gsc@scirp.org 